\title{
Lactoferrin and Neonates: Role in Prevention of Neonatal Sepsis and Necrotizing Enterocolitis
}

\section{Deepak Sharma*, Aakash Pandita and Chetan Kumar}

Department of Neonatology, Fernandez Hospital, Hyderabad, India

*Corresponding author: Deepak Sharma, Department of Neonatology, Fernandez Hospital, Hyderabad, India, Tel: +919462270002; E-mail: dr.deepak.rohtak@gmail.com

Rec date: 12 Oct 2014; Acc date: 15 Oct 2014; Pub date: 17 Oct 2014

Copyright: (C) 2014 Sharma D et al. This is an open-access article distributed under the terms of the Creative Commons Attribution License, which permits unrestricted use, distribution, and reproduction in any medium, provided the original author and source are credited.

\section{Introduction}

Lactoferrin (LF) is a member of the transferring family with a molecular weight of $78-\mathrm{kDa}$ and is present in numerous external secretions including human milk, saliva, tears, airway mucus, and the secondary granules of neutrophils [1]. It is an iron-binding glycoprotein found in abundance in premature milk with its concentration decreasing as the term age [2]. This protein has been establish to cause a number of biological roles, including antimicrobial (Escherichia coli, Staphylococcus aureus, Klebsiella pneumoniae, Streptococcus mutans, Pseudomonas aeruginosa, Haemophilus influenzae, Helicobacter pylori, Clostridium difficile, Shigella Flexnieria) anti-fungal (Candida albicans), anti-cancer (head and neck squamous cell carcinoma), anti-viral (HCV, HIV) antioxidant, and immunomodulatory effects [3]. Partial degradation of LF by pepsin enzyme give rise to peptides termed lactoferricin (LFcin) with most potent antimicrobial action [4].

Neonatal sepsis and Necrotizing enterocolitis (NEC) are very dreadful condition seen in nurseries and as neonatologist are saving more premature and extremely low birth weight infants, the fortunes of these conditions have increased [5]. Neonatal sepsis is a worldwide public health problem, with higher incidence in the evolving world. Infections are often linked to severe late Neuro-developmental impairment and are the main reason of destruction in pre-term infants and a major peril of poor results in these pre-term infants [6]. NEC is a devastating bowel disease affecting more or less $7 \%$ of very-low-birthweight $(<1500 \mathrm{~g}, \mathrm{VLBW})$ infants. These both condition create a considerable load on the health expenditure of the country and prolongs the stay of the infants in the baby's room. Many affairs have been evaluated to prevent or bring down the incidence of sepsis and NEC including probiotics, breast milk, prophylactic antibiotics, aggressive enteral and parenteral nutrition, fluconazole prophylaxis and Lactoferrin [7].

The interest of neonatology in lactoferrin evolved from the first trial done by Manzoni et al. in 2009 [8]. From there many trials have been conducted in nursery showing the good effects of Lactoferrin in the prevention of neonatal sepsis and NEC.

Manzoni et al. in there randomized trial enrolled 472 VLBW infants and these babies were assessed until discharge for the development of sepsis. The intervention infants were randomly assigned to receive orally administered Bovine lactoferrin (BLF) (100 mg/d) alone $(\mathrm{n}=153)$, BLF plus Lactobacillus rhamnosus GG (LGG) $\left(6 \times 10^{9}\right.$ colonyforming units/d) $(\mathrm{n}=151)$, or placebo $(\mathrm{n}=168)$ from birth until day 30 of life (day 45 for neonates $<1000 \mathrm{~g}$ at birth). The main result of the survey was first episode of late-onset sepsis (LOS) which was defined as sepsis occurring more than 72 hours after birth with isolation of any pathogen from blood or from peritoneal or cerebro-spinal fluid. The outcomes of the survey were very bright with an incidence of LOS significantly reduced in the BLF and BLF plus LGG groups $(9 / 153$ [5.9\%] and $7 / 151$ [4.6\%], respectively) when compared with the control group receiving placebo (29/168 [17.3\%]) (RR, 0.34; 95\% CI, $0.17-0.70 ; \mathrm{P}=0.002$ for $\mathrm{BLF}$ vs. control and $\mathrm{RR}, 0.27 ; 95 \% \mathrm{CI}$, $0.12-0.60 ; \mathrm{P}<0.001$ for BLF plus LGG vs. control). The writers concluded that compared with placebo, BLF supplementation alone or in combination with LGG reduced the incidence of a first episode of late-onset sepsis in VLBW neonates [8]. The secondary analysis of the study by Manzoni et al. Demonstrated that, even though the incidence of fungal colonization was comparable (17.6\% in BLF group, $16.6 \%$ in BLF plus the LGG group, and $18.5 \%$ in placebo group) but the incidence of invasive fungal infection (IFI) was significantly decreased in BLF and BLF plus LGG group $(0.7 \%$ and $2.0 \%$, respectively) compared with the placebo group (7.7\%); and this was significantly true both in extremely low birth weight and low birth weight infant. Hence the writers concluded that prophylactic oral administration of BLF reduced the incidence of IFI in preterm VLBW neonates [9].

Ocha et al., in their study enrolment included 190 infants weighing less than $2500 \mathrm{~g}$ at birth. BLF and maltodextrin (placebo) were turned over entirely at $200 \mathrm{mg} / \mathrm{d}$ in 3 divided doses over the first 4 weeks of life. There was decreasing trend in incidence of sepsis in the BLF group [12/95 (12.6\%)] compared to the placebo group [22/95 (23.2\%)]. For babies weighing less than $1500 \mathrm{~g}$ at birth, the occurrence of NEC was $20 \%$ in the BLF group (8/40) versus $40 \%$ in the control group $(16 / 40)$. Though the survey did not have statistical significance, but the reduction of NEC in the LF group was roughly 50\% [10].

Manzoni et al. in their recently well conducted randomized clinical trial bovine lactoferrin supplementation for prevention of necrotizing enterocolitis in very-low-birth-weight neonates enrolled 743 VLBW neonates and they were assessed until discharge for development of NEC. Infants were randomly allotted to receive orally either BLF (100 milligram/day) alone (group LF; $\mathrm{n}=247$ ) or with LGG (at $6 \times 109 \mathrm{CFU} /$ day; group BLF + LGG; $n=238$ ), or placebo (Control group; $n=258$ ) from birth until day 30 of life (45 for neonates $<1000 \mathrm{~g}$ at birth). The main outcome was $\geq$ stage 2 NEC; death-and/or $\geq$ stage 2 NEC prior to dispatch. They reported significantly lower incidence of NEC in groups of BLF and BLF + LGG [5/247 (2.0\%)] and 0/238 (0\%), respectively] than in controls $[14 / 258(5.4 \%)](\mathrm{RR}=0.37 ; 95 \% \mathrm{CI}$ : $0.136-1.005 ; \mathrm{p}=0.055$ for BLF vs. control; $R R=0.00 ; \mathrm{p}<0.001$ for $B L F+$ LGG vs. control). The incidence of death-and/or NEC was significantly lower in both treatment groups $(4.0 \%$ and $3.8 \%$ in BLF and BLF + LGG vs. $10.1 \%$ in control; $\mathrm{RR}=0.39 ; 95 \% \mathrm{CI}: 0.19-0.80$; $\mathrm{p}=0.008$. $\mathrm{RR}=0.37 ; 95 \% \mathrm{CI}: 0.18-0.77 ; \mathrm{p}=0.006$, respectively). The authors concluded that compared with placebo, BLF supplementation alone or in combination with LGG reduced the incidence of $\geq$ stage 2 NEC and of death-and/or $\geq$ stage 2 NEC in VLBW neonates [11]. 
Citation: Sharma D, Pandita A, Kumar C (2014) Lactoferrin and Neonates: Role in Prevention of Neonatal Sepsis and Necrotizing Enterocolitis. J

Page 2 of 2

Kaur et al., in the first trial from our country conducted a randomized control trial. They enrolled 121 low birth weight (less 2000 grams) neonates. BLF ( $\mathrm{n}=59$ ) was supplemented daily from first to 28th day of life and the control group $(n=62)$ received placebo daily from first to 28th day of life. The authors reported that incidence of first episode of culture proven LOS was significantly lower in the BLF group than in the placebo group [2/59 (3.4\%) versus 9/62 (14.5\%); $\mathrm{p}=0.033$. The sepsis attributable mortality after 72 hours of life was comparable among the BLF supplemented and placebo group [0/59 (0) versus $5 / 62(8.1 \%) ; \mathrm{p}=0.058][12]$.

In a recently published randomized control trial Akin et al. assessed the use of oral lactoferrin to prevent nosocomial sepsis and necrotizing enterocolitis of premature neonates and effect on T-regulatory cells. The neonates either VLBW or born before 32 weeks were randomized to receive either placebo $(n=25)$, or $200 \mathrm{mg}$ BLF $(n=25)$ daily throughout hospitalization. The main outcome was episodes of culture proven nosocomial sepsis and NEC. There were fewer sepsis episodes in BLF treated infants (4.4 vs. 17.3/1,000 patient days, $\mathrm{p}=0.007$ ) with none developing NEC, without statistical significance. The authors concluded that LF prophylaxis reduced nosocomial sepsis episodes [13].

These all studies show a beam of hope that lactoferrin may be a silver bullet for the prevention of neonatal sepsis and NEC. In a recently published review Sharma et al. highlighted the various mechanism of action and other beneficial effects of lactoferrin for neonates [14]. Many works are going on around the globe, whose answers may yield us more evidence for the good effects of lactoferrin. Few registered clinical trials which are being conducted includes effect of prebiotic or lactoferrin supplementation in formula on the gut flora of preterm infants, ISRCTN71737811; study of talactoferrin oral solution for nosocomial infection in preterm infants NCT00854633; supplementation with lactoferrin in preterm newborns (lactoprenew), NCT01172236; lactoferrin for prevention of neonatal sepsis (NEOLACTO), NCT01264536; Lactoferrin Infant Feeding Trial (LIFT) to prevent sepsis and death in preterm infants, ACTRN12611000247976; lactoferrin for prevention of sepsis in infants (NEOLACTO), NCT01525316; trial of lactoferrin for prevention of infections in very premature babies (LACUNA), ISRCTN66482337; oral lactoferrin supplementation for prevention of sepsis in preterm neonate, NCT01821989; Enteral Lactoferrin In Neonates (ELFIN), ISRCTN88261002 [4].

\section{References}

1. Iyer S, Lönnerdal B (1993) Lactoferrin, lactoferrin receptors and iron metabolism. Eur J Clin Nutr 47: 232-241.

2. Hirai Y, Kawakata N, Satoh K, Ikeda Y, Hisayasu S, et al. (1990) Concentrations of lactoferrin and iron in human milk at different stages of lactation. J Nutr Sci Vitaminol (Tokyo) 36: 531-544.

3. Orsi N (2004) The antimicrobial activity of lactoferrin: current status and perspectives. Biometals 17: 189-196.

4. Turin CG, Zea-Vera A, Pezo A, Cruz K, Zegarra J, et al. (2014) Lactoferrin for prevention of neonatal sepsis.Biometals 27: 1007-1016.

5. Neu J, Walker WA (2011) Necrotizing enterocolitis. N Engl J Med 364: 255-264.

6. Liu L, Johnson HL, Cousens S, Perin J, Scott S, et al. (2012) Global, regional, and national causes of child mortality: an updated systematic analysis for 2010 with time trends since 2000. Lancet 379: 2151-2161.

7. Grave GD, Nelson SA, Walker WA, Moss RL, Dvorak B, et al. (2007) New therapies and preventive approaches for necrotizing enterocolitis: report of a research planning workshop. Pediatr Res 62: 510-514.

8. Manzoni P, Rinaldi M, Cattani S, Pugni L, Romeo MG, et al. (2009) Bovine lactoferrin supplementation for prevention of late-onset sepsis in very low-birth-weight neonates: a randomized trial. JAMA 302: 1421-1428.

9. Manzoni P, Stolfi I, Messner H, Cattani S, Laforgia N, et al. (2012) Bovine lactoferrin prevents invasive fungal infections in very low birth weight infants: a randomized controlled trial. Pediatrics 129: 116-123.

10. Ochoa TJ, Cam L, Lianos R (2012) Lactoferrin for prevention of sepsis in Peruvian neonates. Pediatric Academic Societies web site.

11. Manzoni P, Meyer M, Stolfi I, Rinaldi M, Cattani S, et al. (2014) Bovine lactoferrin supplementation for prevention of necrotizing enterocolitis in very-low-birth-weight neonates: a randomized clinical trial. Early Hum Dev 90: 60-65.

12. Kaur G, Gathwala G (2013) Efficacy of bovine lactoferrin supplementation in preventing late onset sepsis in low birth weight neonates:a randomised placebo controlled clinical trial. Annual Convention of National Neonatology Forum meeting. NNF India.

13. Akin IM, Atasay B, Dogu F, Okulu E, Arsan S, et al. (2014) Oral Lactoferrin to Prevent Nosocomial Sepsis and Necrotizing Enterocolitis of Premature Neonates and Effect on T-Regulatory Cells. Am J Perinatol.

14. Sharma D, Murki A, Murki S, Pratap OT (2014) Use of lactoferrin in the newborn: where do we stand? J Matern Fetal Neonatal Med9: 1-5. 